\title{
Effects of age on noninvasive assessments of vascular function in nonhuman primates: implications for translational drug discovery
}

Delvin R Knight Jr, Andrew H Smith, Richard L Schroeder, Chunli Huang, David A Beebe, Sharon A Sokolowski and Miao Wang ${ }^{*}$

\begin{abstract}
Background: Endothelium-dependent flow mediated dilation (FMD) and pulse-wave velocity (PWV), are used as measures of vascular health and predictors of cardiovascular risk in clinical studies, and both are age-dependent. Numbers of circulating endothelial microparticles (EMPs) and endothelial progenitor cells (EPCs) are also associated with cardiovascular risk, but independent of age in humans. The use of these measurements for pre-clinical assessment of drug cardiovascular safety and efficacy in non-human primates (NHPs) may promote the translation of drug-induced effects on vascular function to clinic outcomes. However, in NHPs, the age effects on the non-invasive measurements of FMD and PWV and the relationship of EMPs/EPCs with FMD are unknown.

Methods: A non-invasive, clinically-relevant approach to assess FMD and PWV was used to examine their relationship with age and with EMPs/EPCs in NHPs. The effects on FMD of nicotine and rosiglitazone were evaluated in senescent primates in an effort to validate our FMD method for pre-clinical assessment of vascular function.

Results: FMD and PWV methods were established in a colony $(n=25)$ of metabolically healthy, cynomolgus monkeys ranging in age from 6 to 26 years. FMD, defined as the percent change, at 1 min of cuff release, from baseline vascular diameter $(0.15 \pm 0.03 \mathrm{~cm})$, had a strong, negative correlation with age $(r=-0.892, p<0.0001)$, ranging from $6 \%$ to $33 \%$. PWV positively correlated with age $(r=0.622, p<0.002)$ in the same healthy monkeys. Nicotine and rosiglitazone, were evaluated in subsets of senescent primates (mean age $16.3 \pm 1.5[$ SEM] years). Rosiglitazone significantly improved FMD $(21.0 \pm 1.6 \%$ vs. vehicle $16.3 \pm 1.6 \%, p<0.01)$ without changing baseline diameters, and coincided with a significant increase in circulating numbers of endothelial progenitor cells (CD45-CD31 + CD34 + VEGFR2+ $7.1 \pm 1.3$ vs. $4.8 \pm 1.1$ counts $/ \mu \mathrm{l})$ and a decrease in endothelial microparticles (CD45-CD42a-CD54+ $26.7 \pm 11.1 \mathrm{vs.} 62.2 \pm 9.8$ counts/ $\mu \mathrm{l})$ $(p<0.05)$. Conversely, FMD was significantly reduced with nicotine $(8.7 \pm 1.4 \%$ vs. vehicle $20.1 \pm 2.2 \%, p<0.05)$.
\end{abstract}

Conclusions: Adult NHPs demonstrate the characteristic linear relationship between age and vascular function using the non-invasive clinically-related measurements of FMD and PWV. However, numbers of circulating EMPs and EPCs did not correlate with age. Endothelial function assessed with FMD, together with EMPs/EPCs assessment, may serve as a novel approach for translational research and therapeutic discovery. Age should be considered in the study design or data analyses when FMD or PWV is used in NHPs.

Keywords: Flow-mediated dilation, Pulse-wave velocity, Endothelium, Drug discovery, Thiazolidinedione, Endothelial progenitor cell, Endothelial microparticles, Nicotine

\footnotetext{
* Correspondence: wangmiao9@gmail.com

Pfizer Worldwide Research and Development, Cardiovascular and Metabolic

Diseases Research Unit, Pfizer Inc, Groton, CT 06340, USA
}

\section{Biomed Central




\section{Background}

Clinically, vascular function may be evaluated noninvasively by measuring flow mediated vascular dilation (FMD) [1], which assesses endothelial function as arterial vasodilation induced by an increase in luminal blood flow, and pulse wave velocity (PWV), which measures arterial stiffness as the velocity of pressure pulse waves through the aorta [2]. Both endothelial dysfunction and aortic stiffness are associated with diseases and conventional cardiovascular risk factors [1-6]. FMD and PWV are also predictors of cardiovascular events in humans $[7,8]$. Aging is associated with both functional and structural changes in the vasculature [9], and leads to impaired FMD [6] and increased PWV [3,10]. When assessing vascular function during interventional drug trials $[11,12]$, age is a classic confounding factor.

Non-human primate (NHP) is an important translational preclinical model for cardiovascular disease, diabetes and other metabolic diseases [13-16] and like humans can naturally develop glucose intolerance and diabetes without pharmacological or surgical inducement $[16,17]$. FMD and PWV have been used to evaluate test drugs and disease effects $[16,18]$ in NHPs. However, the age effects on these measures of vascular function (FMD and PWV), which are well-documented in humans, remain unaddressed in NHPs. Since NHP can live as adults for decades, a better understanding of the relationship between age and FMD/PWV would be important in order to avoid the confounding effects of age when interpreting FMD and PWV [18].

Thus, our primary goal was to develop the methods to measure FMD and PWV in NHPs and to examine the effects of age on these clinically relevant assessments of vascular function. In addition, we explored using FMD in senescent NHPs as a translational approach to cardiovascular efficacy/safety assessment. Here, we focus on FMD because there is limited information on using PWV for clinical assessment of drug effects, and FMD has emerged as an important clinical measurement for assessing cardiovascular risk factors and disease therapies $[5,11,19]$. For example, chronic treatment with rosiglitazone improve FMD from $\sim 5 \%$ to $\sim 7 \%$ in patients with type 2 diabetes mellitus [11]. To make the efficacy/ safety assessment, rosiglitazone, which targets PPAR $\gamma$ nuclear receptor, was used to show beneficial vascular effects, and nicotine was used to mimic the well-known detrimental effects of smoking on FMD. Finally, endothelial microparticles (EMPs, membrane coated vesicles) and endothelial progenitor cells (EPCs, a population of cells in the blood involved in endothelial repair), are associated with cardiovascular risk in humans [20,21] and are being investigated as clinical biomarkers of endothelial vascular health. Here, we have a unique opportunity to examine the relationship of EPCs/EMPs with age and FMD by addressing these "clinically relevant" biomarkers in our primate model. Since PPAR $\gamma$ improves endothelial functions [22,23] and increases the numbers of EPCs [24,25], we determined the effects of rosiglitazone on circulating EMPs and EPCs, as well as, FMD in NHPs.

\section{Methods}

\section{Animals}

Studies were conducted with the approval of the Pfizer Institutional Animal Care and Use Committee. Twentyfive healthy monkeys (Macaca fascicularis, also known as cynomolgus) (aged at 6 to 26 years, 18 males and 7 females) were studied. These monkeys were paired housed, when appropriate, in a room with temperature of $19-25^{\circ} \mathrm{C}$, humidity of $30-70 \%$, and $12: 45 / 11: 15$ light/ dark cycles with a 45 minute ramping period to full lights on/off as simulated dawn/dusk. The monkeys were fed a primate chow diet (PMI Certified Hi Fiber Primate Diet, LabDiet 5K91, PMI Nutrition International, St. Louis, MO) supplemented with fruits and/or vegetables with the amount adjusted to maintain a normal body weight. Conscious primates, previously conditioned to chair restraint (Plas-Labs, Inc. Lansing, MI), were seated comfortably in a primate chair for drug administration and blood collection prior to vascular measurements.

\section{Measurement of artery flow mediated dilation (FMD) and pulse-wave velocity (PWV)}

We established clinically-relevant, non-invasive methods to examine FMD and PWV in non-human primates (Figure 1). FMD and PWV were measured between 9 am to $12: 30 \mathrm{pm}$ on overnight fasted monkeys. Monkeys were sedated with butorphanol $(0.3 \mathrm{mg} / \mathrm{kg}$, im $)$ and midazolam $(0.5 \mathrm{mg} / \mathrm{kg}$, im), or with ketamine $(5 \mathrm{mg} / \mathrm{kg}, \mathrm{im})+$ midazolam $\left(0.35 \mathrm{mg} / \mathrm{kg}\right.$, im), placed on a $37^{\circ} \mathrm{C}$ heating pad to maintain body temperature, oriented in a right lateral recumbent position to allow access the right brachial and femoral arteries. Stable mean arterial blood pressure and heart rate during these procedures averaged 79 $\mathrm{mmHg}$ and 142 beats/min, respectively.

Electrocardiography (ECG) electrodes were placed, to collect a lead II ECG signal. Pulse waves from the brachial and femoral arteries, were recorded using a SphygmoCor (AtCor, Itasca, IL) PWV probe that was associated with a modified SphygmoCor program (CvMSV9; modified for high rates). Distal-proximal distances were measured and entered into the program. PWV was calculated by the program using the equation PWV $=\Delta x / \Delta t$, where $\Delta x$ is difference in distance between the two sites relative to the sternal notch, and $\Delta t$ is the time for the wave to travel that distance (the difference in time lapse from $\mathrm{R}$-wave peak to pressure wave peak, between the two sites). A minimum of 2 measurements were used to calculate average PWV. 


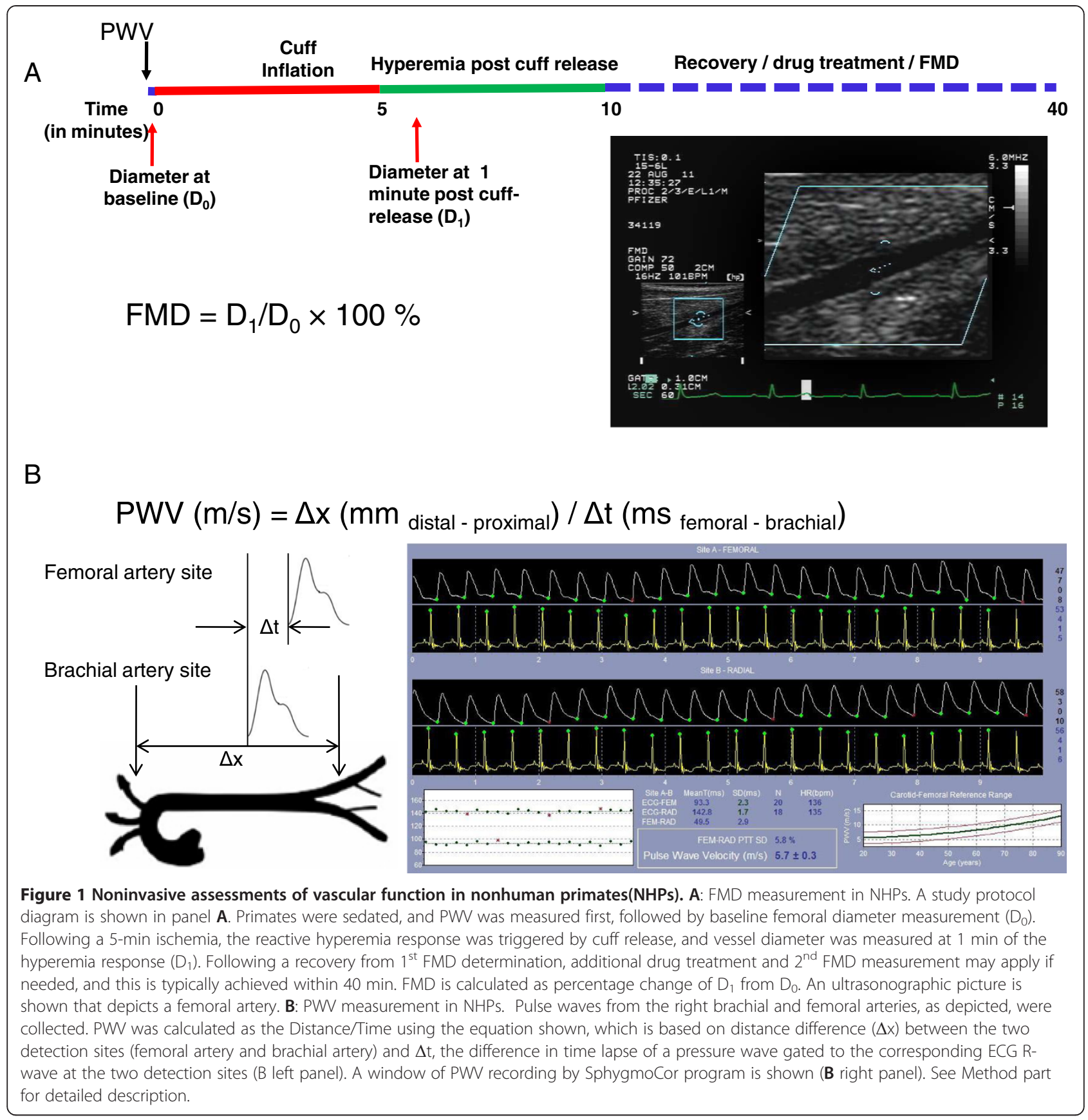

Brachial artery measurements were taken at the axilla and femoral artery measurements were taken mid-thigh for PWV. The average distal distance (femoral to notch) was $338 \pm 5.4 \mathrm{~mm}$ and average proximal distance (brachial to notch) was $75 \pm 3.1 \mathrm{~mm}(\mathrm{n}=25)$. The distal vs. proximal distance allowed sufficient difference to calculate the PWV. Average detection variation for PWV was $10.6 \%$.

FMD in the NHPs was measured using ultrasonography, as illustrated in the Figure 1A. The right leg was positioned and gently restrained to prevent movement during the study. An infant V-Lok cuff (WA Baum,
Copiague, NY) was then placed below the knee and connected to a manometer. After applying ultrasonic gel, a linear array probe (6-15 MHz), connected to a Hewlett Packard Sonos ${ }^{\circledR} 5500$ and mounted on an articulated arm (PPM100, Tektronix, Inc. Beaverton, OR), was positioned over the femoral artery, manipulated until a clear signal was obtained and then locked in place for the remainder of the protocol. Once a baseline recording of the femoral artery was collected, the cuff was inflated within $2 \mathrm{~s}$ to a super-systolic pressure (290-300 $\mathrm{mmHg}$ ). After 5 min the cuff pressure was rapidly released $(<1 \mathrm{sec}$.) 
and a second recording was collected at 1 minute of reperfusion approximating the time of maximal flow mediated dilation [16]. Vessel diameter was measured, using electronic calipers, for baseline and 1 minute recordings. The average baseline femoral artery diameter for the study colony was $0.151 \pm 0.03 \mathrm{~cm}(\mathrm{n}=25)$. Flow mediated dilation was calculated as the $\%$ change from baseline diameter. Each diameter measurement was the average of 4 segmental measurements taken $\sim 1 \mathrm{~mm}$ apart gated to the ECG T wave. At the end of the experiment, monkeys were recovered by naloxone $(0.05 \mathrm{mg} / \mathrm{kg}$ $\mathrm{im}$ and $0.05 \mathrm{mg} / \mathrm{kg}$ iv - if they received butorphanol) and flumazenil ( $2 \mathrm{~mL}$ per monkey, iv). FMD conducted 15 min apart showed average variation $<5.5 \%$.

\section{Study design}

Six fasted monkeys ( $17 \pm 1$ years of age) were treated orally with both vehicle and rosiglitazone $(1 \mathrm{mg} / \mathrm{kg}$, coated on one PrimaTreat; Bio-Serv, Frenchtown, NJ) $\geq 2$ weeks apart in a $2 \times 2$ crossover design ( 2 in vehicle-drug sequence and 4 in drug-vehicle sequence). Drug or vehicle was administered at 6:00 AM, and, FMD and PWV were measured six hours later, a time point that showed increased FMD in a human study [12]. Blood samples were collected (via cephalic vein) just prior to sedation, and immediately processed for determination of endothelial microparticle, endothelial progenitor cell and TNF $\alpha$ (detailed in following methods). These treatments were repeated in the same animals to determine the effect of rosiglitazone on lipids and intravenous glucose tolerance test.

A separate group of monkeys ( $n=3,15 \pm 4$ years of age) were sedated, and intravenously infused with saline vehicle, then with $0.844 \mu \mathrm{g} / \mathrm{kg} / \mathrm{min}$ nicotine bitartrate (Sigma Aldrich, St. Louis, MO), at rate of $0.4 \mathrm{~mL} / \mathrm{min}$. FMD was recorded at $5 \mathrm{~min}$ of vehicle infusion, and at $15 \mathrm{~min}$ of nicotine infusion (while the nicotine infusion was constantly maintained), and the study was completed within 35 min. The nicotine dose used was calculated to approximate the predicted blood nicotine concentration $(40 \mathrm{nM})$ of a human study in which chewing nicotine gum reduced FMD [26,27].

\section{Endothelial microparticle (EMP) and endothelial progenitor cell (EPC) sample preparation and analysis}

To measure EPC, whole blood was collected into EDTA tubes from animals fasted overnight and, incubated with anti-human CD34, CD31, CD45 (Becton Dickinson and Co./BD, Franklin Lakes, NJ) and VEGFR2 (R\&D Systems; Minneapolis, MN.) antibodies. After lysing the red blood cells, the samples were analyzed using a high performance Canto II flow cytometer (BD) with DIVA software (BD).

At the same time, whole blood was collected in sodium citrate tubes and platelet poor plasma (PPP) was prepared to measure EMPs. An aliquot of PPP was added to each of the two panels of antibodies: Panel 1 [CD42a-APC (conjugated in house), CD31-FITC, CD45-AF700, Annexin VV450, CD105-PerCP (BD), CD54-PE (Invitrogen)]; Panel 2 [CD42a-APC (conjugated in house), CD31-FITC, CD45AF700, CD106-PE-Cy7 (conjugated in house), CD62E-PE (BD), VEGFR2-PerCP (R\&D Systems), CD144-Pacific Blue (conjugated in house) (BioLegend, San Diego, CA.)]. Cytofix was then added and a $500 \mathrm{uL}$ aliquot was transferred into a Trucount tube (BD) and analyzed by flow cytometry.

\section{Biochemical tests and cytokine determination}

Venous blood samples were collected in serum separator tubes from untreated, rosiglitazone treated or vehicle treated monkeys fasted overnight, centrifuged at $3000 \mathrm{rpm}$ for $10 \mathrm{~min}$ at $4^{\circ} \mathrm{C}$, and levels of lipids and glucose measured on Siemens Advia 2400 chemistry analyzer, and levels of insulin measured on the Siemens Centaur Immunoassay, with kits of Advia Chemistry Systems (Siemens Healthcare Diagnostics Inc., Tarrytown, NY).

Lipopolysaccharide (LPS, Sigma-Aldrich) stimulated release of TNF $\alpha$ was carried out, ex vivo, using sodium heparin anti-clotted whole blood that was harvested 6 hours after rosiglitazone dosing. Blood was stimulated with $10 \mathrm{ng} / \mathrm{mL}$ LPS for six hours with gentle rocking, plasma separated by $14,000 \mathrm{rpm}$ centrifugation for $20 \mathrm{~min}$ at $10-15^{\circ} \mathrm{C}$, and TNF $\alpha$ measured by using Linco Luminex ${ }^{\circledR}$ mouse cytokine assay(EMD Millipore, Billerica, MA).

\section{Intravenous glucose tolerance test (IVGTT)}

Monkeys fasted overnight were sedated with ketamine for duration of IVGTT. Two iv catheters were placed for blood sampling and glucose administration, respectively. Baseline samples were drawn starting approximately $10 \mathrm{~min}$ after initial ketamine dose and catheter placement. A 50\% dextrose solution was then administered, iv, at $250 \mathrm{mg} / \mathrm{kg}$ over 20 s. Blood samples $(\sim 1 \mathrm{~mL})$ for glucose and insulin analysis were collected in serum tubes at 2, 5, 7, 10, 15, 20 and 30 min post dextrose administration, centrifuged and the resulting serum analyzed for glucose/insulin levels. The Area Under the Curve (AUC) for plasma glucose and insulin levels from 0 to $30 \mathrm{~min}$ was calculated. Glucose disappearance rate was expressed as $K_{\mathrm{glc}}$, the reduction of the $\log _{\mathrm{e}}$ of plasma glucose levels between 5 \& $20 \mathrm{~min}$ following iv glucose administration and calculated using the formula: $K_{\text {glc }}=\left(\log _{e} G_{1}-\log _{e} G_{2}\right) /\left(t_{2}-t_{1}\right)$ where $G_{1}$ and $G_{2}$ are the glucose levels at time $5 \mathrm{~min}\left(t_{1}\right)$ and $20 \mathrm{~min}$ $\left(t_{2}\right)[28]$.

\section{Statistical analysis}

Pearson correlation coefficient was computed for correlation study. Linear regression models were applied to investigate the relationships of age with FMD and PWV and the relationship between FMD and PWV. In order to 
evaluate the treatment effect of rosiglitazone on the endpoints of interest (FMD and PWV) compared to vehicle, a linear mixed effect model was applied to analyze each of the two endpoints from this $2 \times 2$ crossover study with age adjustment. Specifically, the model has treatment (drug vs. vehicle), sequence ( 1 vs. 2 ), and period ( 1 vs. 2 ) as fixed effects, age as a covariate, and subject (monkey) as random effect. Paired $t$ test was used to compare EMPs/EPCs preand post- rosiglitazone treatment. Paired $t$ test was applied to analyze the FMD data measured in the nicotine infusion study to evaluate the effect of nicotine on FMD compared to saline vehicle. Results are presented as parameter estimate (Mean) \pm SEM. A two-sided $\mathrm{p}<0.05$ was considered statistically significant.

\section{Results}

Age correlates to FMD and PWV in NHPs

To examine age effects on FMD and PWV in NHPs, we established clinically-relevant, non-invasive methods
(Figure 1). FMD was measured in a colony of metabolically healthy monkeys $(\mathrm{n}=25)$ with age ranging from 6-26 years. Serum levels of lipids (total cholesterol, HDL, LDL, and triglycerides; Additional file 1: Table S1A) showed no indication of hyperlipidemia, and levels of blood urea nitrogen and creatinine were normal indicating normal renal function. Since cynomolgus monkeys can naturally become glucose intolerant the primates were assessed using an IVGTT. Fasted blood glucose levels were normal and the areas under the curve for glucose and the disappearance of glucose in response to dextrose challenge were also normal, indicating no glucose intolerance in these monkeys (Additional file 1: Table S1B).

In these metabolically healthy monkeys, FMD demonstrated a robust dynamic range (6 to $33 \%$ ) that correlated negatively with age $(\mathrm{r}=-0.892, \mathrm{p}<0.0001$; regression equation: $\mathrm{y}(\% \mathrm{FMD})=-1.119 \mathrm{x}($ year $)+36.00)$ (Figure 2A). These data indicate that age has a significant impact on FMD in NHPs. In the same monkeys,
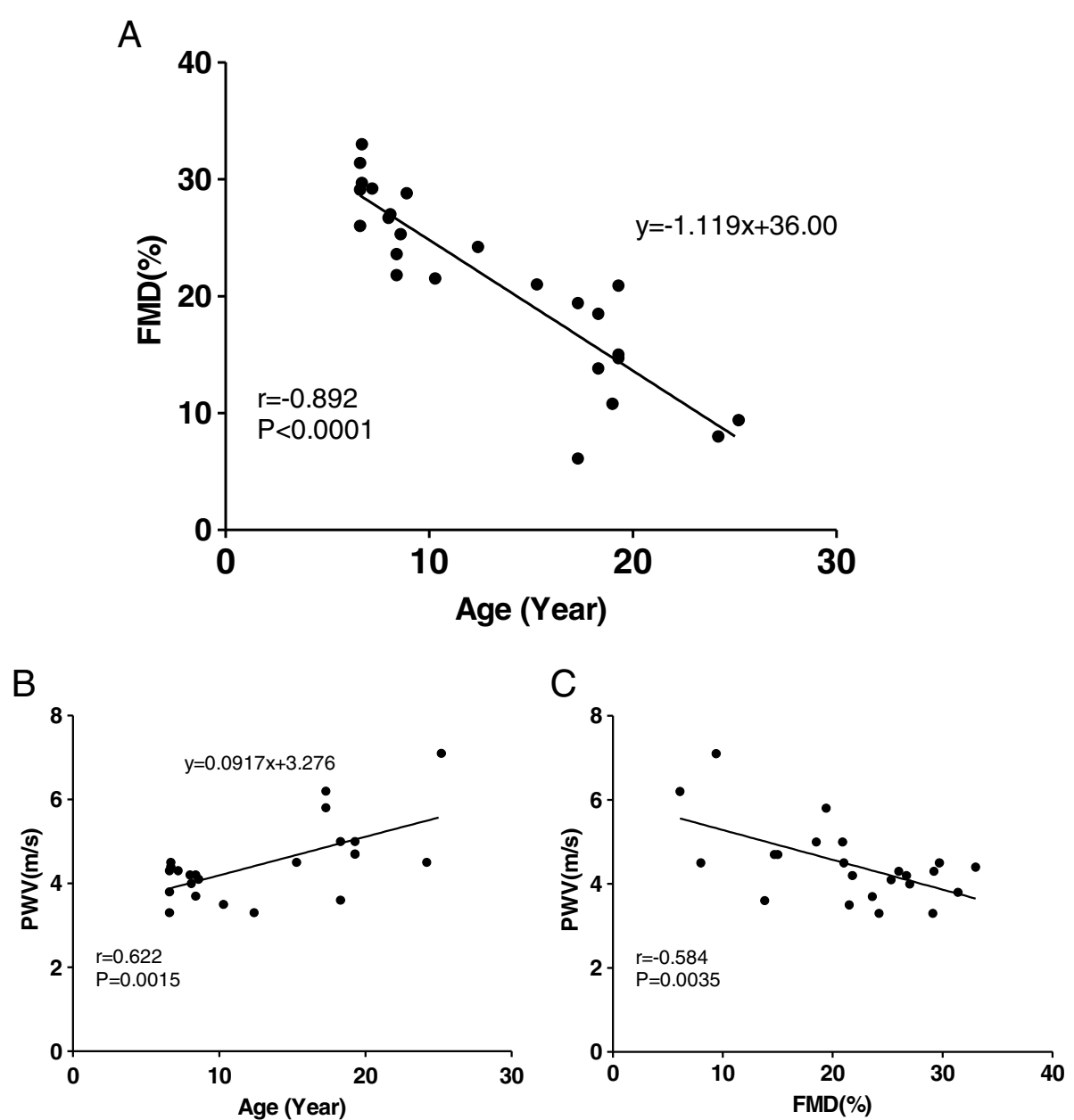

Figure 2 Age correlates to FMD and PWV in NHPs. Age strongly correlates with FMD $(\mathbf{A}: r=-0.892, p<0.0001 n=25)$ and with PW (B: $r=0.622$, $p<0.002, n=23)$. FMD negatively correlates with PW $(\mathbf{C}: r=-0.584, p<0.002, n=23)$. Two monkeys were excluded from the PWW analysis: one with a poor positional signal and one that was (low) out of range. 
PWV ranged from 3 to $7 \mathrm{~m} / \mathrm{s}$, and also correlated with age $(\mathrm{r}=0.622, \mathrm{p}<0.002$; regression equation: $\mathrm{y}(\mathrm{PWV})=$ $0.0917 x($ year $)+3.276)$ (Figure $2 \mathrm{~B})$. The reduction in FMD also correlated with increase in $\operatorname{PWV}(\mathrm{r}=-0.584$, $\mathrm{p}<0.002$ ) (Figure 2C). However, unlike FMD and PWV, age did not significantly correlate with numbers of EPCs or subsets of EMPs in these monkeys (Additional file 2: Table S2).

Rosiglitazone improved, while nicotine impaired, FMD in metabolically healthy monkeys

Using senescent monkeys, we examined the effect of rosiglitazone on FMD. The FMD was significantly increased following a single oral dose of rosiglitazone $(21.0 \pm$ $1.6 \%$ vs. vehicle $16.3 \pm 1.6 \%$ ) (Figure 3 ). Rosiglitazone treatment had no effect on baseline femoral artery diameter $(1.52 \pm 0.04$ vs. $1.51 \pm 0.04 \mathrm{~mm}$, respectively), and did not affect PWV (Additional file 3: Figure S1) when compared to vehicle treatment. In addition, when compared to vehicle treatment, this dose of rosiglitazone had no significant metabolic effects on baseline glucose, insulin, lipids, IVGTT responses, or anti-inflammatory effects, as indicated by the lack of LPS-stimulated TNF $\alpha$ release in these metabolically healthy primates (Additional file 4: Table S3).
However, rosiglitazone treatment significantly increased EPCs (CD45-CD31 + CD34 + VEGFR2+ $7.1 \pm 1.3$ vs. $4.8 \pm$ 1.1 counts/ $\mu \mathrm{l})$ and reduced EMPs (CD45- CD42a- CD54+ $26.7 \pm 11.1$ vs. $62.2 \pm 9.8$ counts $/ \mu \mathrm{l}$ ) (Additional file 3 : Figure S2). CD45- CD42a- CD105 + EMPs (16.5 \pm 3.7 vs. $33.2 \pm 5.6$ counts/ $\mu \mathrm{l}, \mathrm{n}=5, \mathrm{p}=0.06)$ and CD45- CD42aCD144+EMPs $(5.0 \pm 1.5$ vs. $41.8 \pm 17.6$ counts $/ \mu \mathrm{l}, \mathrm{n}=5$, $\mathrm{p}=0.09)$ also trended lower.

We next examined whether the deleterious effect of nicotine on FMD can be detected in NHPs. Intravenous infusion of nicotine in healthy monkeys significantly reduced FMD $(8.7 \pm 1.4 \%$ vs. vehicle $20.1 \pm 2.2 \%)$ (Figure 4$)$. The plasma level of nicotine, at the time of FMD measurement, was $\sim 27 \mathrm{nM}$.

\section{Discussion}

Non-invasive measurement of vascular function in NHPs We established a non-invasive method for FMD to examine the effects of age on vascular function in NHPs. Our protocol to measure FMD in NHP was adapted from human protocols $[1,7,29,30]$ and as such, FMD was measured following a hyperemic response triggered by distal limb ischemia. An important aspect of the present technique was the use of a combination of anesthetics
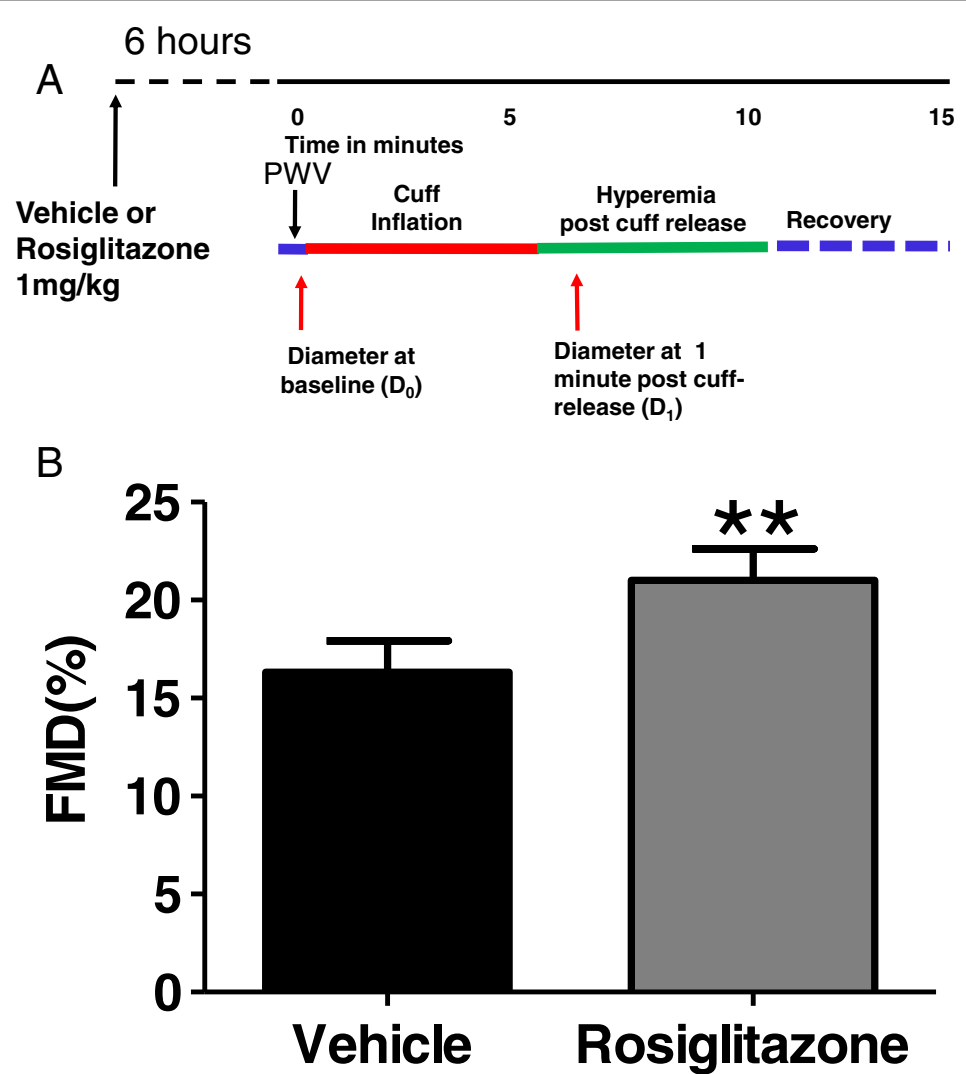

Figure 3 Acute treatment of rosiglitazone (panel A, study protocol, see Methods for details) improves FMD in NHPs (panel B). Results were adjusted by age. $n=6 ;{ }^{* *}: p<0.01$. 


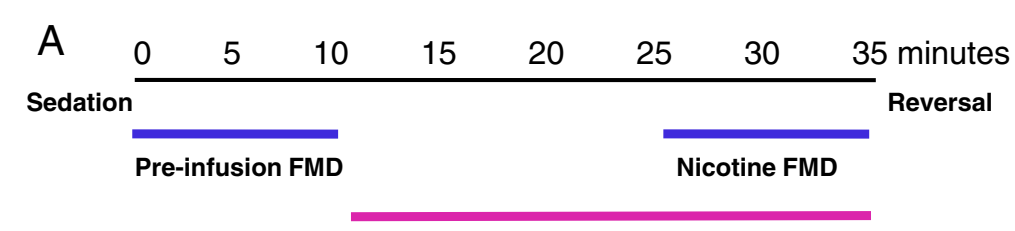

Nicotine bitartrate $0.844 \mathrm{ug} / \mathrm{kg} / \mathrm{min}, 0.4 \mathrm{ml} / \mathrm{min}$

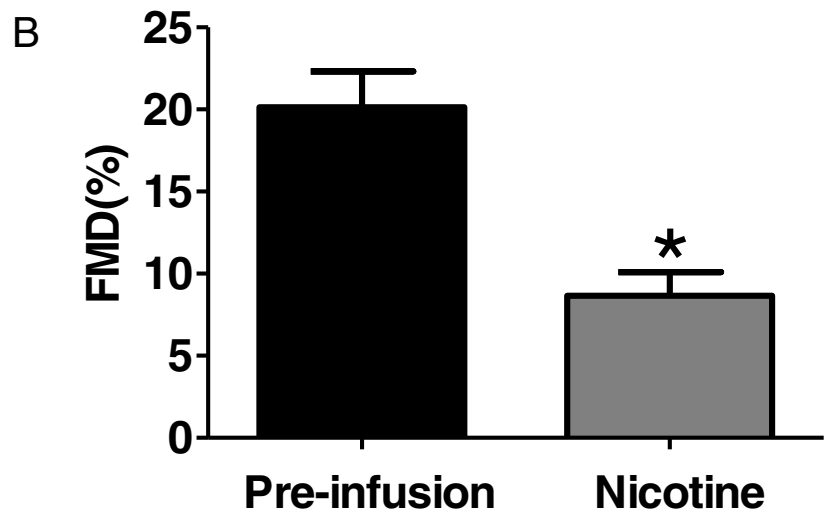

Figure 4 Nicotine infusion (panel A, study protocol, see Methods for details) impairs FMD in NHPs (panel B). $n=3 ;{ }^{*}: p<0.05$.

(midazolam and butorphanol or ketamine) to keep the muscle relaxed without limb movement that was not observed with ketamine alone. Measuring FMD using the articulating arm also obviates the need to manipulate the probe while measuring vascular diameter. These refinements were key in allowing us to detect a dynamic range of changes in FMD associated with age and pharmacological interventions in NHPs.

\section{Age and FMD}

Previously, total peripheral resistance was shown to fall less with acetylcholine infusion in $19.8 \pm 0.6$ years old monkeys than in $7.1 \pm 0.4$ years old monkeys [31], suggesting an age-related impairment of vascular dilatory response to acetylcholine. Since NHP can live as adults for decades, a better understanding of the relationship between age and FMD/PWV would be important in order to avoid the confounding effects of age. Here, we examined the age effect on the flow-induced vascular dilatory response in a colony of adult, sexually mature monkeys, 6 to 26-years of age. The robust age-FMD relationship (Figure 2A) has at least three implications. (i) The agedependent nature of FMD recapitulates that seen in human [6], suggesting a translational feature of NHP model of FMD. (ii) For studies evaluating the effects of test agents on FMD in NHPs, prior randomization by age and/ or recording of age at baseline is necessary, and agedependent vs. disease-ameliorating effects on FMD may also need to be considered to clarify mechanisms of action. (iii) Prior vascular health assessment by FMD can be used to select NHPs most likely affected by test agents.
This is particularly important for selecting study subjects from a heterogeneous population of NHPs. For example, monkeys develop diabetes and metabolic syndrome at varied age $[16,17]$, and diabetes itself features endothelial dysfunction (reduced FMD) in human [32]. In addition, the age-FMD relationship allows us to put in perspective the effect of test agents on vascular function (FMD). It is interesting to note that the average improvement in FMD following rosiglitazone treatment (Figure 3) was equivalent to improving the monkeys' vascular age by 5.2 years.

PWV also correlates with age in NHPs (Figure 2), indicating that, similar to the FMD assessment, age effect should also be considered when evaluating PWV, by prior randomization of study groups or by age adjustment. Furthermore, age-adjustment decreases variation in FMD and PWV by $79.57 \%$ and $38.67 \%$, respectively, among these NHPs. This suggests a stronger age impact on FMD than PWV.

\section{Evaluation of FMD changes in NHPs}

We established that the non-invasive methodology can be used to assess beneficial (rosiglitazone) and detrimental (nicotine) FMD changes following drug intervention in NHPs (Figures 3 and 4). Chronic treatment of diabetic patients with rosiglitazone ameliorates insulin resistance and its related metabolic disorder while improving FMD [11]. Acute treatment also results in FMD improvement in healthy subjects in the absence of associated metabolic effects [12]. In line with these earlier studies, we reported here that rosiglitazone improved FMD in metabolically healthy NHPs (Figure 3), without drug-related 
metabolic or anti-inflammatory effects (Additional file 4: Table S3). In a recent study [16] using NHPs of similar age to those studied here, 6-week pioglitazone treatment improves metabolic parameters, and reverses the impaired FMD that co-emerged with the metabolic syndrome. In senescent euglycemic monkeys, our results show that rosiglitazone caused an acute improvement in FMD that was independent of effects on cytokine (TNFo) release or metabolic effects on glucose and lipids. This suggests that PPAR $\gamma$ agonists may have a direct action on the endothelium in vivo beyond its systemic metabolic effects.

Despite the lack of age-related correlation of EPCs and any of the EMPs, acute treatment with rosiglitazone decreased the number of EMP(CD45-CD42a-CD54+) and increased EPC(CD45-CD31 + CD34 + VEGFR2+) (Additional file 3: Figure S2). CD54(ICAM-1) is an intercellular adhesion molecule expressed on endothelial cells, and is associated with increased cell activation [33]. Although somewhat controversial, EPCs are believed to play an important role in the endogenous repair mechanisms for maintaining the integrity of the endothelial monolayer [34,35]. PPAR $\gamma$ is known to regulate endothelial survival and function [22,23], as well as, EPC function [24,25]. The rosiglitazone induced increase in FMD coincided with acute increases in EPCs and suppression of EMPs suggesting an improvement in endothelial health. While these changes in EPCs and EMPs do not provide a causal, mechanistic link for the FMD changes, from a drug discovery view point, our results could provide the impetus for further studies to evaluate the use of EPCs/EMPs as biomarkers to potentially capture a signal of vasculature change of test drugs.

Cigarette smoking is associated with impairment of FMD $[1,36,37]$, and treatment with nicotine gum attenuates FMD in human subjects [26]. In this study, the plasma levels of infused nicotine $(\sim 27 \mathrm{nM})$, at the time of FMD measurement, matched to the levels in humans that are associated with reduced FMD [37], and also approximated the nicotine level $(\sim 40 \mathrm{nM})$ predicted from another human study, in which nicotine gum treatment reduces FMD [26]. Nicotine's effect on the cardiovascular system is complex [38], including release of proinflammatory mediators, thrombosis, decreasing oxygen supply, and increasing sympathetic nerve activity [39]. The significant reductions in FMD with nicotine further support the translation of this model to clinical outcomes and illustrates how robust this preclinical, NHP model is in detecting changes in FMD (only 3 animals required) in a highly controlled laboratory setting.

Unlike other models [40], the NHP is considered as a highly translational animal model of cardiovascular disease [13], and is often used as a preclinical model to evaluate therapeutic agents. This translation is also supported by the observed correlation between age and
FMD/PWV in this study. NHPs are phylogenetically closer to humans, and may be particularly useful when a drug target does not exist in other species (for example, Cholesteryl ester transfer protein, a regulator of HDL levels, is not present in rodents). The noninvasive nature of FMD allows reuse of animals for cross-over and longitudinal studies. Further, the pharmacological effects on FMD might be detected with a relatively small group of animals (for example, 6 monkeys were used in the rosiglitazone study) under a well-controlled environment that is difficult to duplicate in clinical studies. The described FMD methodology using NHPs, might be highly valuable both for translational research, drug efficacy/safety evaluation, and for clinical dose-selection. This is particularly important for developing diabetes drugs. New guidelines of FDA require cardiovascular safety assessment before approval of any diabetes drugs [41].

\section{Study limitations}

Blood flow was not assessed during these FMD determinations. FMD measured under our current protocol does not distinguish between macro- and micro-circulation effects; rather, it serves as an integrated assessment of vascular endothelium function. The variability of PWV detection under current protocol needs to be optimized, which may limit the power to detect a biological impact. Detection of EMPs and EPCs may have been limited by the availability of antibodies and the degree of crossspecies reactivity of the human antibodies with monkey. As they become available, the use of additional antibodies for EMP/EPC markers and their functional characterization could provide further insight and utility in assessing vascular health.

\section{Conclusions}

In conclusion, we established a non-invasive, clinicallyrelevant approach to assess vascular function in NHP by measuring FMD and PWV, and identified age as a significant modifier of FMD, and to a less degree, of PWV. Acute treatment with rosiglitazone improves FMD, while nicotine reduces FMD in senescent primates. Senescent primates with age-related endothelial dysfunction may provide a unique model for evaluating preclinically the efficacy and safety of therapeutic agents on endothelial function (i.e. FMD). The endothelial function assessment with FMD in NHPs and the associated circulating biomarkers (i.e. EPC, EMP), as described herein, may serve as a novel approach for translational research and therapeutic discovery. Like in humans, age is an important confounder that should be considered when assessing cardiovascular health and interpreting drug actions in NHPs. 


\section{Additional files}

Additional file 1: Table S1. Basal metabolic parameters of the study monkeys. A: Blood lipids profile. B: Glucose, insulin, and IVGTT.

Additional file 2: Table S2. FMD or age does not correlate with numbers of EPCs or EMPs in metabolically healthy monkeys. $n=19$ and 21 for EPC and EMP, respectively.

Additional file 3: Figure S1. Rosiglitazone does not affect PW in NHPs. $n=6, p=0.22$ (age adjusted). NS: non-significant. Figure S2. Rosiglitazone results in favorable changes in circulating numbers of EMPs (CD45-CD42a-CD54+) (A) and EPCs (CD45-CD31 + CD34 + VEGFR2+) (B) in euglycemic, senescent monkeys. $n=5$, * $p<0.05$; **: $p<0.01$.

Additional file 4: Table S3. Effect of rosiglitazone treatment on blood biochemistry and LPS-stimulated TNFa release. No significant difference between the two groups, $n=5$ (vehicle) or 6 (rosiglitazone). $K_{\mathrm{glc}}, 5-20$ : slope of the disappearance of glucose 5-20 min after dosing; $\mathrm{AUC}_{0-30}$ : area under the curve for glucose or insulin (0-30 min).

\section{Abbreviations}

FMD: Flow mediated dilation; PWV: Pulse-wave velocity; NHP: Non-human primate; EMP: Endothelial microparticle; EPC: Endothelial progenitor cell; PPARY: Peroxisome proliferator-activated receptor gamma; IVGTT: Intravenous glucose tolerance test.

\section{Competing interests}

The authors declare that they have no competing interests. DRK's current address is National Institutes of Health, Department of Health and Human Services. The opinions expressed in this article are the authors' own and do not reflect the view of the National Institutes of Health, the Department of Health and Human Services, or the United States government.

\section{Authors' contributions}

DRK designed and supervised the study while employed at Pfizer, Inc., and RLS and AHS performed the in vivo study. MW designed the nicotine study and the cytokine study. DAB and MW assisted with the in vivo study setup and sample processing. SAS and $\mathrm{CH}$ designed the EMP and EPC analysis, $\mathrm{CH}$ measured the EMPs and EPCS, and SAS analyzed and interpreted the EMP and EPC data. DRK, AHS, and MW analyzed and interpreted the in vivo and in vitro data. MW wrote the manuscript. All authors have read and approved the manuscript for publication.

\section{Acknowledgements}

We appreciate Harshan Pisharath (Clinical Veterinary Science, Pfizer Inc.) for help on setting up the ultrasound-imaging based FMD measurement. We thank Jeffrey Chabot (PDM, Pfizer Inc.)for nicotine dose selection, Angela C Wolford (PDM, Pfizer Inc.) for nicotine measurement, Yanwei Zhang (DSRD, Pfizer Inc.), Dingzhou Li (Reg Strategy \& Compliance, Pfizer Inc.) for statistical help.

Received: 20 November 2012 Accepted: 9 April 2013

Published: 22 April 2013

\section{References}

1. Celermajer DS, et al: Non-invasive detection of endothelial dysfunction in children and adults at risk of atherosclerosis. Lancet 1992, 340(8828):1111-1115.

2. Wilkinson IB, Cockcroft JR, Webb DJ: Pulse wave analysis and arterial stiffness. J CardiovasC Pharmacol 1998, 32(Suppl 3):S33-S37.

3. Avolio AP, et al: Effects of aging on changing arterial compliance and left ventricular load in a northern Chinese urban community. Circulation 1983, 68(1):50-58.

4. Halcox JP, et al: Endothelial function predicts progression of carotid intima-media thickness. Circulation 2009, 119(7):1005-1012

5. Charakida M, et al: Assessment of atherosclerosis: the role of flowmediated dilatation. Eur Heart J 2010, 31(23):2854-2861.

6. Celermajer DS, et al: Endothelium-dependent dilation in the systemic arteries of asymptomatic subjects relates to coronary risk factors and their interaction. J Am Coll Cardio/ 1994, 24(6):1468-1474.
7. Yeboah J, et al: Brachial flow-mediated dilation predicts incident cardiovascular events in older adults: the Cardiovascular Health Study. Circulation 2007, 115(18):2390-2397.

8. Vlachopoulos C, Aznaouridis K, Stefanadis C: Prediction of cardiovascular events and all-cause mortality with arterial stiffness: a systematic review and meta-analysis. J Am Coll Cardiol 2010, 55(13):1318-1327.

9. Lakatta EG: Cardiovascular regulatory mechanisms in advanced age. Physiol Rev 1993, 73(2):413-467.

10. Mattace-Raso FU, et al: Determinants of pulse wave velocity in healthy people and in the presence of cardiovascular risk factors: 'establishing normal and reference values'. Eur Heart J 2010, 31(19):2338-2350.

11. Kelly AS, et al: Rosiglitazone improves endothelial function and inflammation but not asymmetric dimethylarginine or oxidative stress in patients with type 2 diabetes mellitus. Vasc Med 2007, 12(4):311-318.

12. Walcher $T$, et al: Rapid effect of single-dose rosiglitazone treatment on endothelial function in healthy men with normal glucose tolerance: data from a randomised, placebo-controlled, double-blind study. Diab Vasc Dis Res 2010, 7(3):178-185

13. Shelton KA, Kaplan TBCJR, Nonhuman Primate Models of Atherosclerosis: Nonhuman Primates in Biomedical Research, Volume Chapter 8. Secondth edition; 2012:385-411.

14. McLenachan JM, et al: Loss of flow-mediated endothelium-dependent dilation occurs early in the development of atherosclerosis. Circulation 1991, 84(3):1273-1278.

15. Howard TD, et al: Epigenetic changes with dietary soy in cynomolgus monkeys. PLoS One 2011, 6(10):e26791.

16. Zhang $X$, et al: Rhesus macaques develop metabolic syndrome with reversible vascular dysfunction responsive to pioglitazone. Circulation 2011, 124(1):77-86.

17. Wagner JD, et al: Naturally occurring and experimental diabetes in cynomolgus monkeys: a comparison of carbohydrate and lipid metabolism and islet pathology. Toxicol Pathol 2001, 29(1):142-148.

18. Farrar DJ, et al: Anatomic correlates of aortic pulse wave velocity and carotid artery elasticity during atherosclerosis progression and regression in monkeys. Circulation 1991, 83(5):1754-1763.

19. Moens $\mathrm{AL}$, et al: Flow-mediated vasodilation: a diagnostic instrument, or an experimental tool? Chest 2005, 127(6):2254-2263.

20. Werner $\mathrm{N}$, et al: Circulating endothelial progenitor cells and cardiovascular outcomes. N Engl J Med 2005, 353(10):999-1007.

21. Bernal-Mizrachi $L$, et al: High levels of circulating endothelial microparticles in patients with acute coronary syndromes. Am Heart J 2003, 145(6):962-970.

22. Kanda $T$, et al: PPARgamma in the endothelium regulates metabolic responses to high-fat diet in mice. J Clin Invest 2009, 119(1):110-124.

23. Werner $C$, et al: Pioglitazone activates aortic telomerase and prevents stress-induced endothelial apoptosis. Atherosclerosis 2011, 216(1):23-34

24. Liang $C$, et al: Rosiglitazone via upregulation of Akt/eNOS pathways attenuates dysfunction of endothelial progenitor cells, induced by advanced glycation end products. Br J Pharmacol 2009, 158(8):1865-1873.

25. Sorrentino SA, et al: Oxidant stress impairs in vivo reendothelialization capacity of endothelial progenitor cells from patients with type 2 diabetes mellitus: restoration by the peroxisome proliferator-activated receptor-gamma agonist rosiglitazone. Circulation 2007, 116(2):163-173.

26. Rudolph TK, et al: Myeloperoxidase deficiency preserves vasomotor function in humans. Eur Heart J 2011, 33(13):1625-1634.

27. Hukkanen J, Jacob P 3rd, Benowitz NL: Metabolism and disposition kinetics of nicotine. Pharmacol Rev 2005, 57(1):79-115.

28. Jen K-LC, Hansen BC: Glucose disappearance rate in rhesus monkeys: Some technical considerations. Am J Primatol 1988, 14(2):153-166.

29. Rossi $R$, et al: Prognostic role of flow-mediated dilation and cardiac risk factors in post-menopausal women. J Am Coll Cardiol 2008, 51(10):997-1002.

30. Gaenzer $\mathrm{H}$, et al: Flow-mediated vasodilation of the femoral and brachial artery induced by exercise in healthy nonsmoking and smoking men. J Am Coll Cardiol 2001, 38(5):1313-1319.

31. Asai $K$, et al: Peripheral vascular endothelial dysfunction and apoptosis in old monkeys. Arterioscler Thromb Vasc Biol 2000, 20(6):1493-1499.

32. Feng $B$, et al: Circulating level of microparticles and their correlation with arterial elasticity and endothelium-dependent dilation in patients with type 2 diabetes mellitus. Atherosclerosis 2010, 208(1):264-269.

33. Jimenez JJ, et al: Endothelial cells release phenotypically and quantitatively distinct microparticles in activation and apoptosis. Thromb Res 2003, 109(4):175-180. 
34. Urbich C, Dimmeler S: Endothelial progenitor cells: characterization and role in vascular biology. Circ Res 2004, 95(4):343-353.

35. Foteinos $\mathrm{G}$, et al: Rapid endothelial turnover in atherosclerosis-prone areas coincides with stem cell repair in apolipoprotein E-deficient mice. Circulation 2008, 117(14):1856-1863

36. Celermajer DS, et al: Cigarette smoking is associated with dose-related and potentially reversible impairment of endothelium-dependent dilation in healthy young adults. Circulation 1993, 88(5 Pt 1):2149-2155.

37. Kallio K, et al: Tobacco smoke exposure is associated with attenuated endothelial function in 11-year-old healthy children. Circulation 2007, 115(25):3205-3212.

38. Glantz SA, Parmley WW: Passive smoking and heart disease. Mechanisms and risk. JAMA 1995, 273(13):1047-1053.

39. Najem B, et al: Acute cardiovascular and sympathetic effects of nicotine replacement therapy. Hypertension 2006, 47(6):1162-1167.

40. Libby P, Ridker PM, Hansson GK: Progress and challenges in translating the biology of atherosclerosis. Nature 2011, 473(7347):317-325.

41. Center for Drug Evaluation and Research, F.a.D.A: Guidance for Industry, Diabetes Mellitus - Evaluating Cardiovascular Risk in New Antidiabetic Therapies to Treat Type 2 Diabetes. www.fda.gov/downloads/Drugs/GuidanceCompliance RegulatoryInformation/Guidances/UCM071627.pdf, 2008.

doi:10.1186/1479-5876-11-101

Cite this article as: Knight et al:: Effects of age on noninvasive assessments of vascular function in nonhuman primates: implications for translational drug discovery. Journal of Translational Medicine 2013 11:101.

\section{Submit your next manuscript to BioMed Central and take full advantage of:}

- Convenient online submission

- Thorough peer review

- No space constraints or color figure charges

- Immediate publication on acceptance

- Inclusion in PubMed, CAS, Scopus and Google Scholar

- Research which is freely available for redistribution 